\title{
Silicon Heterostructures as High Performance Field Effect Transistor
}

\author{
G. K. Yegon \\ ${ }^{I}$ (Department of Mathematics and Physics, Moi University, Kenya)
}

\begin{abstract}
The advances in Silicon technology have driven the MOSFET device fabrication towards submicron regime. Despite all these advances in technology special effects come into play such as velocity overshoot, short channel effects and Drain Induced Barrier Lowering (DIBL). For MOSFET with large geometry, they experience a number of effects ranging from low clock frequencies due to high input capacitance, high threshold voltage hence high power consumption and lower trans-conductance. As the dimensions are scaled down, the drain current increases, evidence that sub-micron devices have better performance as compared to un-scaled devices. It can also be noted that there is a strong correlation between device dimensions and device performance. Also from transfer curves the output drain current decreases with increase in the drain voltage but it was further established from the transfer curves that the trans-conductance of the device increases with scaling at a constant voltage. This shows that sub-micron device has better performance as compared to unscaled device.
\end{abstract}

Keywords: MOSFET, short channel effects, velocity overshoot, DIBL, silicon.

\section{Introduction}

A field - effect transistor is a voltage controlled variable resistor. Unlike bipolar transistor, the field effect (unipolar) transistor operates on electric conduction carried by majority carriers. For the structure of a double diffused Field Effect transistor the middle P region serves as a conductive channel [1]. Two ohmic contacts are made to the channel, one acting as a source to supply carriers and the other as the drain to collect carriers. Voltage $\mathrm{V}_{\mathrm{D}}$ is applied between source and drain. For drain made of $\mathrm{P}$ - type semiconductor, the drain is negatively biased with respect to the source [2]. MOSFET scalling is fundamental in improving its performance by enhancing its frequency and lowering power consumption [3].

\section{Theory and Equations}

Considering regions $\mathrm{AB}$ as shown in diagram below, the action of resistance modulation takes place and represents the field effect transistor by an idealized structure.
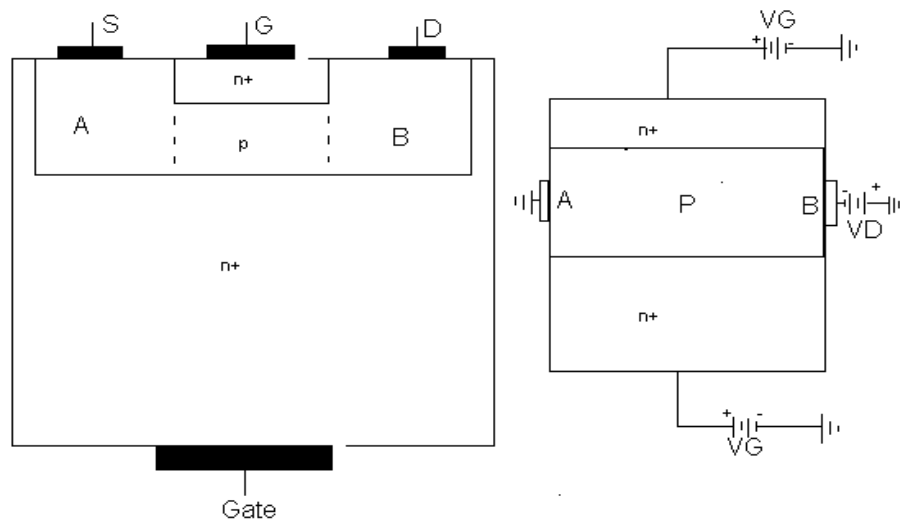

Figure 1. 1 Diagrams showing the representation of field effect transistor by an idealized structure

The middle $\mathrm{P}$ region that forms a conductive channel is only moderately doped as compared to the two $n$ regions; therefore, the depletion (space charge) region extends almost entirely into the channel. As $V_{G}$ or $V_{D}$ or both are increased, depletion region widens, reducing the channel width. The progressive change in effective width of the channel is as shown below [4]. 


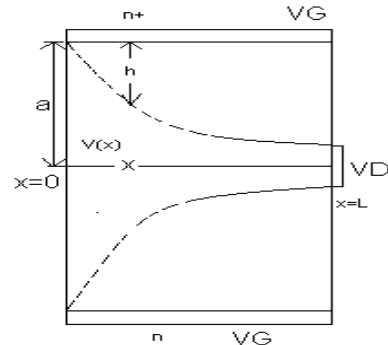

(a)

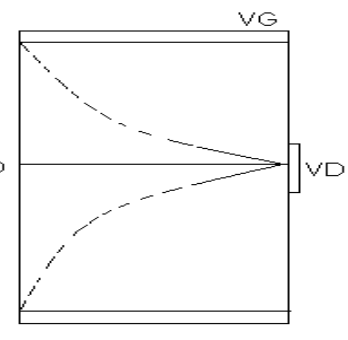

(b)

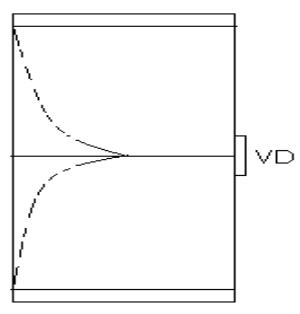

(c)

Figure 1. 2 The progressive change in effective width of the channel with $\mathrm{V}_{\mathrm{G}}$.

The diagrams of figure 2.2 show effect of the gate voltage $\mathrm{V}_{\mathrm{G}}$ on the width of the conduction channel for $\mathrm{V}_{\mathrm{G} 1}, \mathrm{~V}_{\mathrm{G} 2}$ and $\mathrm{V}_{\mathrm{G} 3}$ where $\mathrm{V}_{\mathrm{G} 1}<\mathrm{V}_{\mathrm{G} 2}<\mathrm{V}_{\mathrm{G} 3}$. The conduction channel is bounded by the dashed lines. The channel is pinched off at the drain and the pinch-off point is moved toward the source end as $\mathrm{V}_{\mathrm{G}}$ is progressively increased.

Assuming uniform doping concentration $\mathrm{N}_{\mathrm{a}}$ in the $\mathrm{P}$ region, let $-V(x)$ be voltage at a point $\mathrm{X}$ in the channel.

The depletion depth $\mathrm{h}$ is given by [5]

$h(x)=\left(\frac{2 \in}{e N_{a}}\right)^{\frac{1}{2}}\left[V_{d}+V_{G}+V(x)\right]^{\frac{1}{2}}$

$V_{d}$ is the diffusion (or built in) voltage

$V_{G}$ being positive and $V_{D}$ being negative with respect to the source.

The width of conductive channel is $2(a-h)$ and the hole concentration in $\mathrm{P}-$ region is $\mathrm{N}_{\mathrm{a}}$. Thus current through the channel is $I_{D}=e \mu_{P} N_{a} E_{X} Z[2 a-2 h(x)]$

Where $\dot{Z}$ is the lateral dimension of the transistor structure. Realizing that $E_{x}=-\frac{d}{d x}[-V(x)]$ then,

$$
\int_{x=0}^{L} I_{D} d x=\int_{0}^{V D} 2 e \mu_{p} N_{a} Z[a-h(x)] d V
$$

Substituting (1) into (3) then,

$$
\begin{aligned}
& \int_{x=0}^{L} I_{D} d x=\int_{0}^{V_{D}}\left\{2 e \mu_{p} N_{a} Z\left[a-\left(\frac{2 \in}{e N_{a}}\right)^{\frac{1}{2}}\left(V_{d}+V_{G}+V_{D}\right)^{\frac{1}{2}}\right\} d V\right. \\
& I_{D} L=2 e \mu_{P} N_{a} Z \int_{0}^{V_{D}}\left\{a-\left(\frac{2 \epsilon}{e N_{a}}\right)^{\frac{1}{2}}\left[V_{d}+V_{G}+V_{D}\right]^{\frac{1}{2}}\right\} d V \\
& I_{D}=\frac{2 e \mu_{p} N_{a} Z}{L} \int_{0}^{V_{D}}\left\{a-\left(\frac{2 \in}{e N_{a}}\right)^{\frac{1}{2}}\left[V_{d}+V_{G}+V_{D}\right]^{\frac{1}{2}}\right\} d V \\
& =\frac{2 e \mu_{p} N_{a} Z a}{L}\left\{V_{D}-\left(\frac{2 \in}{e N_{a}}\right)^{\frac{1}{2}} \cdot \frac{2}{3 a}\left[\left(V_{d}+V_{G}+V_{D}\right)^{\frac{3}{2}}-\left(V_{d}+V_{G}\right)^{\frac{3}{2}}\right]\right\}
\end{aligned}
$$




$$
=G_{m}\left\{V_{D}-\left(\frac{2 \epsilon}{e N_{a}}\right)^{\frac{1}{2}} \cdot \frac{2}{3 a}\left[\left(V_{d}+V_{G}+V_{D}\right)^{\frac{3}{2}}-\left(V_{d}+V_{G}\right)^{\frac{3}{2}}\right]\right\}
$$

where $G_{m}=2 e \mu_{P} N_{a} a \frac{Z}{L}$

Equation (4) holds as long as $h(x)<a$ for $0<x<L \quad$ Pinch - off voltage $V_{p}^{\prime}$ is defined such that when $V_{G}=V_{p}^{\prime}$ at $\mathrm{x}=0$, the value of $\mathrm{h}(0)=\mathrm{a}$ in equation (1), thus;

$$
h(x)=\left(\frac{2 \epsilon}{e N_{a}}\right)^{\frac{1}{2}}\left\|V_{d}+V_{G}+V_{(x)}\right\|^{\frac{1}{2}}
$$

When $V_{G}=V_{p}^{\prime}$ at $x=0$, the value of $h(0)=a$

$$
\begin{array}{ll}
a=\left(\frac{2 \in}{e N_{a}}\right)^{\frac{1}{2}}\left[V_{d}+V_{p}^{\prime}+0\right]^{\frac{1}{2}} & a^{2}=\frac{2 \in}{e N_{a}}\left[V_{d}+V_{p}^{\prime}\right] \\
e \frac{1}{2 \in} N_{a} a^{2}-V_{d}=V_{p}^{\prime} & V_{p}^{\prime}=V_{p}-V_{d} \\
V_{p}^{\prime}=\frac{e N_{a}}{2 \in} a^{2}-V_{d}=V_{p}-V_{d} &
\end{array}
$$

Figure 2.2 corresponds to pinch - off at the drain with $V_{G}+V_{D}=V_{p}^{\prime}$. In terms of $V_{p}^{\prime}$, equation (5) becomes $\begin{array}{llll}V_{p}^{\prime}+V_{d}=\frac{e N_{a}}{2 \epsilon} a^{2} & \text { and } \quad \text { substituting } & \text { in } & \text { equation } \\ I_{D}=G_{m}\left\{V_{D}-\frac{2}{3\left(V_{p}^{\prime}+V_{d}\right)^{\frac{1}{2}}}\left[\left(V_{d}+V_{G}+V_{D}\right)^{\frac{3}{2}}-\left(V_{d}+V_{G}\right)^{\frac{3}{2}}\right]\right\} & \end{array}$

Figure 1.2 shows $I_{D}-V_{D}$ characteristics

The numbers for $I_{D}, V_{G}$ and $V_{D}$ shows their magnitudes in typical field - effect transistor.

For low $\mathrm{V}_{\mathrm{D}}$, expansion of $\left(V_{d}+V_{G}+V_{D}\right)^{\frac{3}{2}}$ term in (6) gives (Fariborz,1993)

$$
I_{D}=G_{m}\left[1-\left(\frac{V_{d}+V_{G}}{V_{d}+V_{p}^{\prime}}\right)^{\frac{1}{2}}\right] V_{D}
$$

In this region $\mathrm{I}_{\mathrm{D}}$ increases linearly with $V_{D}$. At $V_{D}=V_{P}^{\prime}-V_{G}$, the current $I_{D}$ becomes saturated. Values of $V_{D}$ and $I_{D}$ at this point are denoted by $V_{D S}$ and $I_{D S}$. Differentiating $I_{D}$ with respect to $V_{D}$, it's found out that;

$$
g_{D}=\frac{\partial I_{D}}{\partial V_{D}}=G_{m}\left[1-\left(\frac{V_{d}+V_{G}+V_{D}}{V_{d}+V_{p}^{1}}\right)^{\frac{1}{2}}\right]
$$

But $g_{D}$ becomes zero when $V_{D}=V_{P}^{\prime}-V_{G}$ i.e. the current remains constant at $V_{D S}$ for any incremental change in $V_{D}$. The quantity $g_{D}$ is called drain conductance. 
The trans-conductance is further defined as

$$
g_{m}=\frac{\partial I_{D}}{\partial V_{G}}=\frac{-G_{m}}{\left(V_{d}+V_{P}^{\prime}\right)^{\frac{1}{2}}}\left[\left(V_{d}+V_{G}+V_{D}\right)^{\frac{1}{2}}-\left(V_{d}+V_{G}\right)^{\frac{1}{2}}\right]
$$

In terms of $g_{D}$ and $g_{m}$, the a.c. component of the drain is

$$
I_{D}=g_{m} V_{G}+g_{D} V_{D}
$$

Where $V_{G}$ and $V_{D}$ is a.c. gate and drain voltages.

\section{Modeling And Simulation}

This study was conducted through device simulation. It was concerned with investigating the effect of scaling MOSFET gate dimensions on the performance of the device. It is specifically intended to investigate the relationship between saturation drain current against saturation drain voltage at different scaling factors. Such issues are best investigated through simulation method. This method enables the researcher to manipulate the gate dimensions and study/observe the resulting effects. This simulation research design entails, inputting the parameters required, systematic manipulation of some characteristics and examination of the outcome. All simulations were done using MATHCAD software. NMOSFET with gate lengths $100 \AA, 65 \AA, 42.25 \AA, 27.27 \AA$ and $17.85 \AA$, gate widths $100 \AA$ and with oxide thickness of $2 \AA$ were studied. A gate length of $100 \AA$ was chosen as a very large device and has been scaled by a factor of $k, k^{2}, k^{3}, k^{4}$ where $k=0.65$. The results obtained were then used to plot the graphs using ORIGIN lab software at. The transfer curves were then obtained at different drain voltages of $1-5 \mathrm{~V}$.

Assuming doping concentration $\mathrm{N}_{\mathrm{a}}$ in the $\mathrm{P}$ substrate region, the gate voltage $\left(\mathrm{V}_{\mathrm{G}}\right)$, drain voltage $\left(V_{D}\right)$, built in voltage $\left(V_{d}\right)$, gate length $(L)$, gate width $(Z)$, electron charge $(e)$ and electron mobility $\left(\mu_{\mathrm{p}}\right)$ then the following equations were used to simulate the results from equation below;

$$
\begin{gathered}
I_{D S}=K V_{D S}^{2}=K\left(V_{G}-V_{T}\right)^{2} \quad \text { Where } \\
K=\frac{\in \mu_{n} z}{2 L a}
\end{gathered}
$$

This gives the change in drain current $\mathrm{I}_{\mathrm{DS}}$,sat (saturation) with the drain voltage $\mathrm{V}_{\mathrm{DS} \text {,sat }}$ at different scaling factors. Also equation below was used to plot graphs of drain current against gate voltage

$I_{D}=\frac{\in \mu_{n} Z}{L d}\left[\left(V_{G}-V_{T}\right) V_{D}-V_{D}^{2}\right]$

\section{Figures And Discussions}

This chapter contains the results of all simulations done for drain current verses drain voltage. Figures 2.1 to 2.3 shows the graphs of drain current against drain voltage at different gate voltage and with different scaling factors.

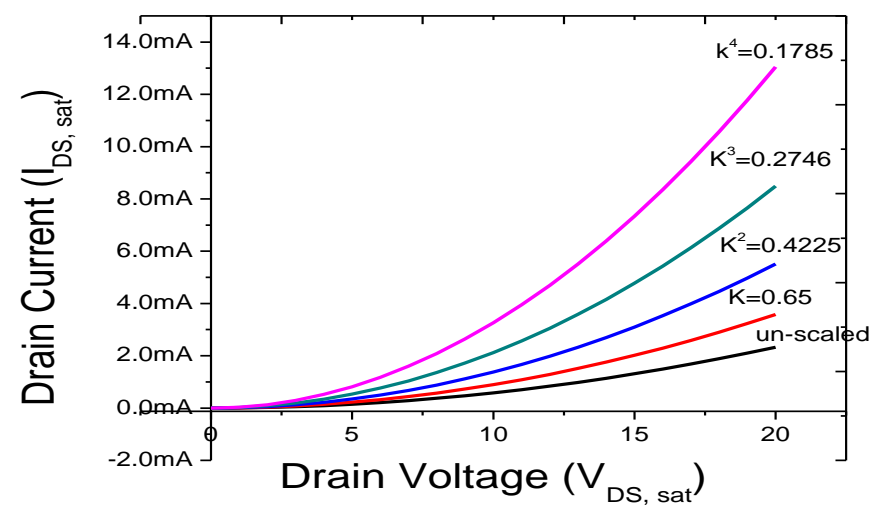

Figure 2.1 Graph of $I_{D S}$, sat $-V_{D S}$, sat at saturation at different scaling factors (un-scaled, $K=0.65, K^{2}=0.4225$, $\mathrm{K}^{3}=0.2746$ and $\mathrm{K}^{4}=0.1785$ ) 
Figure 2.1 shows a graph of saturation drain current against saturation drain voltage. It is observed that for unscaled device, the change in drain current against drain voltage is small. As the device is scaled down, the change in drain current against drain voltage increases.

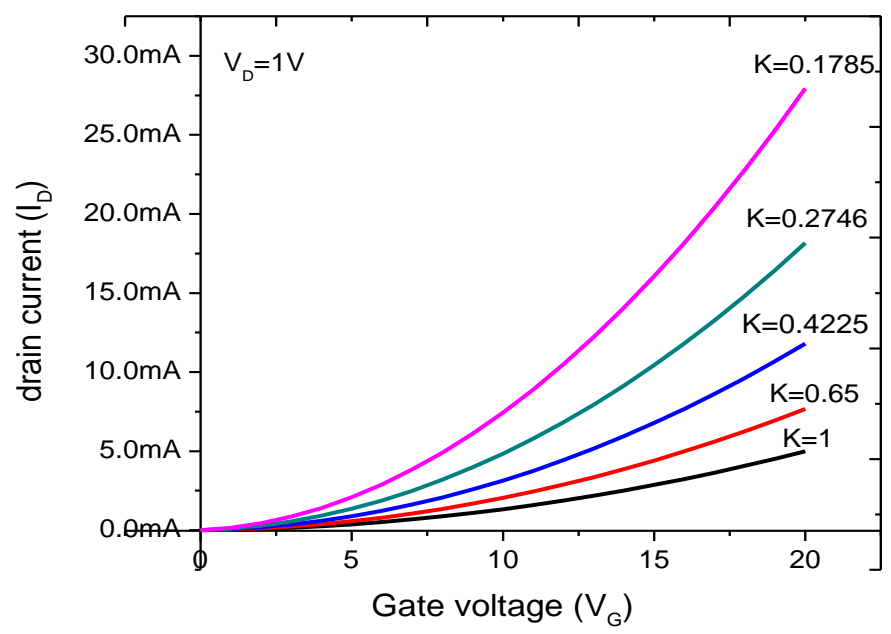

Figure 2.2 Graph of drain current against gate voltage (transfer curves) at $\mathrm{V}_{\mathrm{D}}=1 \mathrm{~V}$ and at different scaling factors (un-scaled, $\mathrm{K}=0.65, \mathrm{~K}^{2}=0.4225, \mathrm{~K}^{3}=0.2746$ and $\mathrm{K}^{4}=0.1785$ )

Figure 2.2 shows a graph of drain current against gate voltage at $\mathrm{VD}=1 \mathrm{~V}$ and at different scaling factors. For un-scaled device at $\mathrm{L}=100 \AA, \mathrm{Z}=100 \AA$ the rate of change of drain current against gate voltage is small but increases with scaling. This is an increase in the trans-conductance of the device with scaling. It's also observed that there is a shift in the threshold voltage. The threshold voltage decreases with scaling.

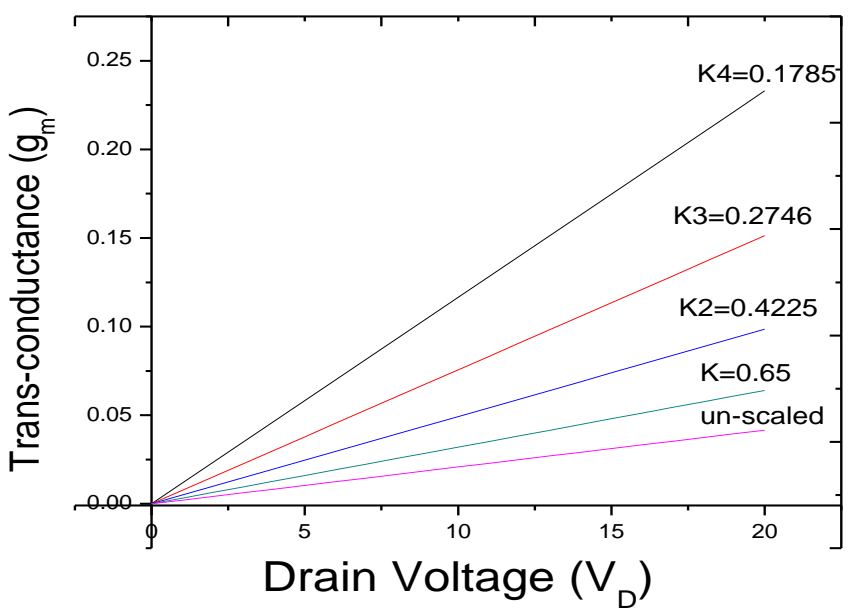

Figure 2.3 Graph of trans-conductance against drain voltage at different scaling factor

Figure 2.3 shows the graph of trans-conductance $\left(g_{m}\right)$ against drain voltage $\left(\mathrm{V}_{\mathrm{D}}\right)$. It's observed that gm is directly proportional to the drain voltage. It's also worth noting that down-scaling the device increases the trans-conductance of the device. The figure above shows the $g_{m}-V_{D}$ graph at various scaling factors as indicated. The smaller the device, the higher the trans-conductance.

\section{Conclusion}

The MOSFET controls the drain current by controlling the population of charge carriers from the nchannel. When the gate is made more positive, it accumulates the majority carriers to a larger zone around the gate and this increases the current flow for a given value of VDS. Therefore, modulating the gate voltage modulates the current flow through the device [6]. This is evident in the characteristic curves for the device. 
The findings indicated that for a miniaturized device there was quite an improvement on its performance. To turn the MOSFET ON the input capacitance $\mathrm{C}_{\text {iss }}$ must be charged as fast as possible. To turn it OFF it involves the discharge of capacitance $\mathrm{C}_{\mathrm{iss}}$. The turn on delay $\mathrm{t}_{\mathrm{d}(\mathrm{on})}$ is the time required to charge the input capacitance to threshold voltage level $\left(V_{T H}\right)$. The rise time, $t_{r}$ is the gate charging time from $V_{T H}$ level to full gate. For small geometry design it's observed that input capacitance is small hence smaller turn on delay and smaller rise time. This owes to the reduction in parasitic capacitances hence a higher speed. It's also observed that for a MOSFET with large geometry the drain current is significantly small but can be increased by scaling down the device. This shows that there is a strong correlation between device dimensions and device performance. The shorter the length, the greater the driving ability because channel resistance is proportional to channel length. As a consequence performance of the MOSFET can be enhanced by downscaling. In-view of all this it requires the industry to intensify research of CMOS into an increasingly difficult manufacturing domain. However, there are many challenges to be faced as device dimensions become smaller, and therefore a method should be developed that will enable to compensate for this short channel effects.

\section{References}

[1] Y.Tsividis and C. McAndrew, Operation and modeling of the MOS transistor (Oxford University Press, New York, $3^{\text {rd }}$ ed., 2011).

[2] S. M., Sze, and k. Ng, Kwok, Physics of Semiconductor Devices (John Wiley \& Sons Inc. $3^{\text {rd }}$ ed., 2007).

[3] Hutagalung, S. D., Scaling CMOS to the Limit, IBM J. Res. Dev. Vol. 46, 2002

[4] M.S., Tyagi, Introduction to Semiconductor Materials and Devices (John Wiley \& Sons, Inc. 1991).

[5] F. Assideraghi et al, IEEE, Electron Device Letters, Vol. 14 no. 6, June, 1993.

[6] E. H., Nicollian and J. R. Brews, MOS (Metal Oxide Semiconductor) Physics and Technology (Wiley, 4 March, 1982).

G. K. Yegon. "Silicon Heterostructures as High Performance Field Effect Transistor." IOSR Journal of Applied Physics (IOSR-JAP) 9.4 (2017): 54-59. 